\title{
ANALYSIS OF AN AGE-DEPENDENT SI EPIDEMIC MODEL WITH DISEASE-INDUCED MORTALITY AND PROPORTIONATE MIXING ASSUMPTION: THE CASE OF VERTICALLY TRANSMITTED DISEASES
}

\author{
M. EL-DOMA
}

Received 30 January 2003 and in revised form 8 February 2004

An SI epidemic model for a vertically as well as horizontally transmitted disease is investigated when the fertility, natural mortality, and disease-induced mortality rates depend on age and the force of infection corresponds to a special form of intercohort transmission called proportionate mixing. We determine the steady states and obtain explicitly computable threshold conditions, and then perform stability analysis.

\section{Introduction}

In this paper, we study an age-structured SI epidemic model, where age is assumed to be the chronological age, that is, the time since birth. The disease is fatal and horizontally as well as vertically transmitted. Horizontal transmission is the passing of infection through direct or indirect contact with infected individuals, for example, malaria is a horizontally transmitted disease. Vertical transmission is the passing of infection from parents to newborn or unborn offspring, for example, AIDS, Chagas, and hepatitis B are vertically (as well as horizontally) transmitted diseases. Vertical transmission plays an important role in maintaining some diseases, for example, see [5, 6]. In [14], several examples of vertically transmitted diseases are given, and [5] is devoted to the study of the models and dynamics of vertically transmitted diseases.

In this paper, we study an SI age-structured epidemic model with vertical transmission and disease-induced mortality rate. We determine the steady states, prove thresholds results, and then perform stability analysis.

For the present model, we show that there is a parameter $R(\alpha)$, where $\alpha(a)$ is the disease-induced mortality rate, which determines the existence of a unique endemic steady state if $R(\alpha)<1<R(0)=R_{0}$. Actually, in this case, a trivial steady state is also a possible steady state, and if $R_{0}<1$, then there is only a trivial steady state. The endemic steady state, under suitable conditions, is locally asymptotically stable whenever it exists; and the trivial steady state is globally stable if $R_{0}<1$ and unstable if $R(\alpha)<1<R_{0}$.

We also show that if $q R(\alpha)>1$, where $q$ is the vertical transmission parameter, see Section 2 for definitions, then the only steady state for the model is the trivial steady 
state. Note that if $q=0$, that is, the case of no vertical transmission, then if $R(\alpha)>1$, the only steady state is the trivial steady state. However, if $R(\alpha)>1$, but $q R(\alpha)<1$, then an endemic steady state as well as a trivial steady state are possible steady states; the endemic steady state may not be unique in this case due to lack of monotonicity.

In addition, we show that if we impose some conditions on the epidemiological and demographic parameters of the model, then it is possible to show that the model could give rise to a continuum of nontrivial endemic steady states. In this respect, this model behaves like the SIR model studied in [8]. Also, by other assumptions, we could obtain that the total population consists of infectives only or susceptibles only. The stability of some of these steady states are determined.

In [4], an SIR age-structured epidemic model with disease-induced mortality rate independent of age, and without vertical transmission, is considered. The analysis was carried out when the death, birth, and recovery rates are constants independent of age. Similar threshold conditions for the existence of the unique endemic equilibrium, when $R(\alpha)<1<R_{0}$, as in this paper are obtained in the special case $q=0$. Furthermore, numerical investigations indicated that the endemic equilibrium is locally asymptotically stable, this is, in agreement with our results in this paper.

In $[12,17]$, a McKendrick-Von Foerster type equation for an SI age-structured epidemic model with disease-induced mortality, but without assuming vertical transmission, is studied. It is a simpler version of the SIR age-structured epidemic model studied in [4]. The steady states are determined and some stability results are given. The present paper generalizes the results of these two papers by including the case of vertical transmission.

In [18], an SI age-structured epidemic model with vertical transmission as well as horizontal transmission is studied when the disease-induced mortality rate is constant, and a fraction of the offspring of infected mothers die of AIDS effectively at birth and the remaining fraction survive. The model is for HIV/AIDS, assuming HIV infection always leads to AIDS. Analytical results as well as numerical examples are given, and in a simple example, it has been shown that $R_{0}>1$ is a requirement for the infection to develop; this is in agreement with our results in this paper. An extension to the model in [18] to an SIR age-structured epidemic model to model HIV/AIDS, assuming HIV infection does not necessarily lead to AIDS, is given in [19]. The asymptotic behavior of the solution is explored and several numerical examples are given. In [1], refinements of the models in $[18,19]$ are discussed.

We note that several recent papers have dealt with age-structured epidemic models with vertical transmission, but without disease-induced mortality, for example, see $[8,10,11,13]$. We observe that in such models, there is a restrictive condition on the epidemiological and demographic parameters of the model for the existence of the endemic equilibrium; for example, a typical condition would be, some quantity equals one, whereas for the models in $[12,17]$ and this paper, we see that there is some parameter range of values for which the endemic equilibrium exists.

We also note that the model we investigate in this paper is based on some restrictive assumptions: we assume that the latent period is negligibly short and the infectivity is independent of the duration of the infection. As has been stated in $[1,3,18,19]$, this 
simplification is a preliminary to numerical works for a more complicated and realistic model. For discussions of other types of models and assumptions, see [2, 7, 15, 16, 21, 22].

The organization of this paper is as follows: in Section 2, we describe the model and obtain the model equations; in Section 3, we reduce the model equations to several subsystems; in Section 4, we determine the steady states; and in Section 5, we perform stability analysis.

\section{The model}

In this section, we consider an age-structured population of variable size exposed to a fatal communicable disease. The disease is both vertically and horizontally transmitted. We assume the following.

(1) $s(a, t)$ and $i(a, t)$, respectively, denote the age-density for susceptibles and infectives of age $a$ at time $t$. Then $\int_{a 1}^{a_{2}} s(a, t) d a=$ total number of susceptibles at time $t$ of ages between $a_{1}$ and $a_{2}$ and $\int_{a 1}^{a_{2}} i(a, t) d a=$ total number of infectives at time $t$ of ages between $a_{1}$ and $a_{2}$.

We assume that the total population consists entirely of susceptibles and infectives.

(2) The horizontal transmission of the disease occurs according to the following proportionate mixing assumption (see Dietz and Schenzle [9]): $k_{1}(a) s(a, t) \int_{0}^{\infty} k_{2}\left(a^{\prime}\right) i\left(a^{\prime}\right.$, t) $d a^{\prime}$, where $k_{1}(a)$ and $k_{2}(a)$ are bounded, nonnegative, continuous functions of $a$, and $k_{2}(a)$ is not identically zero. The term $k_{1}(a) \int_{0}^{\infty} k_{2}\left(a^{\prime}\right) i\left(a^{\prime}, t\right) d a^{\prime}$ is called "force of infection" and we let $\lambda(t)=\int_{0}^{\infty} k_{2}(a) i(a, t) d a$.

(3) The fertility rate $\beta(a)$ is nonnegative and continuous, with compact support $[0, A]$ $(A>0)$. The number of births of susceptibles per unit time is given by $s(0, t)=\int_{0}^{\infty} \beta(a)[s(a$, $t)+(1-q) i(a, t)] d a, q \in(0,1]$, where $q$ is the probability of vertically transmitting the disease;

$$
i(0, t)=q \int_{0}^{\infty} \beta(a) i(a, t) d a,
$$

that is, all newborns from susceptibles are susceptible, but a fraction $q$ of newborns from infected parents are infective, that is, they acquire the disease via birth (vertical transmission).

(4) The natural death rate $\mu(a)$ is the same for susceptibles and infectives and $\mu(a)$ is a nonnegative, continuous function and there exists $a_{0} \in[0, \infty)$ such that

$$
\mu(a)>\bar{\mu}>0 \quad \forall a>a_{0}, \quad \mu\left(a_{2}\right)>\mu\left(a_{1}\right) \quad \forall a_{2}>a_{1}>a_{0}
$$

(5) The disease-induced death rate $\alpha(a)$ is a nonnegative, continuous function of $a \in$ $[0, \infty)$.

(6) The initial age distributions $s(a, 0)=s_{0}(a)$ and $i(a, 0)=i_{0}(a)$ are continuous, nonnegative, and integrable functions of $a \in[0, \infty)$.

These assumptions lead to the following system of nonlinear integro-partial differential equations with nonlocal boundary conditions, which describes the dynamics of the 
transmission of the disease:

$$
\begin{gathered}
\frac{\partial s(a, t)}{\partial a}+\frac{\partial s(a, t)}{\partial t}+\mu(a) s(a, t)=-k_{1}(a) s(a, t) \lambda(t), \quad a>0, t>0 \\
\frac{\partial i(a, t)}{\partial a}+\frac{\partial i(a, t)}{\partial t}+\mu(a) i(a, t)=k_{1}(a) s(a, t) \lambda(t)-\alpha(a) i(a, t), \quad a>0, t>0 \\
s(0, t)=\int_{0}^{\infty} \beta(a)[s(a, t)+(1-q) i(a, t)] d a, \quad t \geq 0 \\
i(0, t)=q \int_{0}^{\infty} \beta(a) i(a, t) d a, \quad t \geq 0 \\
\lambda(t)=\int_{0}^{\infty} k_{2}(a) i(a, t) d a, \quad t \geq 0, \\
s(a, 0)=s_{0}(a), \quad i(a, 0)=i_{0}(a), \quad a \geq 0 .
\end{gathered}
$$

We note that problem (2.3) is an SI epidemic model; the same model but with $q=0$ (the case of no vertical transmission) is dealt with in [12, 17]. Also in [10], problem (2.3) is considered with $\alpha=0$.

\section{Reduction of the model}

In this section, we develop some preliminary formal analysis of problem (2.3). We define $p(a, t)$ by

$$
p(a, t)=s(a, t)+i(a, t) .
$$

Then from (2.3), by adding the equations, we find that $p(a, t)$ satisfies the following:

$$
\begin{gathered}
\frac{\partial p(a, t)}{\partial a}+\frac{\partial p(a, t)}{\partial t}+[\mu(a)+\alpha(a)] p(a, t)=\alpha(a) s(a, t), \quad a>0, t>0, \\
p(0, t)=B(t)=\int_{0}^{\infty} \beta(a) p(a, t) d a, \quad t \geq 0, \\
p(a, 0)=p_{0}(a)=s_{0}(a)+i_{0}(a), \quad a \geq 0 .
\end{gathered}
$$

Also, from (2.3), $s(a, t)$ and $i(a, t)$ satisfy the following systems of equations:

$$
\begin{gathered}
\frac{\partial s(a, t)}{\partial a}+\frac{\partial s(a, t)}{\partial t}+\mu(a) s(a, t)=-k_{1}(a) s(a, t) \lambda(t), \quad a>0, t>0, \\
s(0, t)=\int_{0}^{\infty} \beta(a)[s(a, t)+(1-q) i(a, t)] d a=B(t)-i(0, t), \quad t \geq 0, \\
s(a, 0)=s_{0}(a), \quad a \geq 0, \\
\frac{\partial i(a, t)}{\partial a}+\frac{\partial i(a, t)}{\partial t}+\mu(a) i(a, t)=k_{1}(a) s(a, t) \lambda(t)-\alpha(a) i(a, t), \quad a>0, t>0, \\
i(0, t)=q \int_{0}^{\infty} \beta(a) i(a, t) d a, \quad t \geq 0, \\
i(a, 0)=i_{0}(a), \quad a \geq 0, \\
\lambda(t)=\int_{0}^{\infty} k_{2}(a) i(a, t) d a, \quad t \geq 0 .
\end{gathered}
$$

So, it is clear that (3.2), (3.3), and (3.4) are equivalent to the original problem (2.3). 


\section{The steady states}

In this section, we look at the steady-state solution of problem (2.3). A steady state $s^{*}(a)$, $i^{*}(a), \lambda^{*}$, and $B^{*}$ must satisfy the following equations:

$$
\begin{gathered}
\frac{d s^{*}(a)}{d a}+\mu(a) s^{*}(a)=-\lambda^{*} k_{1}(a) s^{*}(a), \quad a>0, \\
s^{*}(0)=\int_{0}^{\infty} \beta(a)\left[s^{*}(a)+(1-q) i^{*}(a)\right] d a=B^{*}-i^{*}(0), \\
\frac{d i^{*}(a)}{d a}+[\mu(a)+\alpha(a)] i^{*}(a)=\lambda^{*} k_{1}(a) s^{*}(a), \quad a>0, \\
i^{*}(0)=q \int_{0}^{\infty} \beta(a) i^{*}(a) d a, \\
\lambda^{*}=\int_{0}^{\infty} k_{2}(a) i^{*}(a) d a .
\end{gathered}
$$

Anticipating our future needs, we define the threshold parameter $R(\alpha)$ by

$$
R(\alpha)=\int_{0}^{\infty} h(a) d a
$$

where $h(a)$ and other related functions are defined as follows:

$$
\begin{aligned}
h(a) & =\beta(a) \pi_{2}(a), \\
\pi_{2}(a) & =\pi(a) \exp \left(-\int_{0}^{a} \alpha(\tau) d \tau\right), \\
\pi(a) & =\exp \left(-\int_{0}^{a} \mu(\tau) d \tau\right) .
\end{aligned}
$$

Note that $R(\alpha)$ is the expected number of offspring of an infected individual at birth over a life time. Also note that $R(0)$, usually denoted by $R_{0}$, is the net reproduction rate. Thus, $R_{0}$ is the expected number of offspring produced in a life time by an individual in the absence of the disease. For example, if $R_{0}>1$, then the total population is growing and becomes unbounded when $t \rightarrow \infty$. In this paper, we will see that, in some situations, $R_{0}$ may be greater than one but the disease controls the population size and thus, keeps the size bounded.

By solving (4.1), we obtain that $s^{*}(a)$ satisfies

$$
s^{*}(a)=s^{*}(0) \pi(a) \exp \left(-\lambda^{*} \int_{0}^{a} k_{1}(\tau) d \tau\right) .
$$

By using (4.8) in (4.2), we obtain that $i^{*}(a)$ satisfies

$$
i^{*}(a)=i^{*}(0) \pi_{2}(a)+\lambda^{*} s^{*}(0) \pi_{2}(a) \int_{0}^{a} F_{k_{1}}(\sigma) d \sigma,
$$

where $F_{v}(\sigma)$ is given by

$$
F_{v}(\sigma)=v(\sigma) \exp \left(\int_{0}^{\sigma}\left[\alpha(\tau)-\lambda^{*} k_{1}(\tau)\right] d \tau\right) .
$$


Using (4.2) and (4.9), we find that

$$
i^{*}(0) \cdot T=q \lambda^{*} B^{*} \int_{0}^{\infty} \int_{0}^{a} h(a) F_{k_{1}}(\sigma) d \sigma d a,
$$

where $T$ is defined as follows:

$$
T=1-q R(\alpha)+q \lambda^{*} \int_{0}^{\infty} \int_{0}^{a} h(a) F_{k_{1}}(\sigma) d \sigma d a .
$$

From (4.3) and (4.9), we obtain that

$$
\lambda^{*}\left[1-s^{*}(0) \int_{0}^{\infty} \int_{0}^{a} f(a) F_{k_{1}}(\sigma) d \sigma d a\right]=i^{*}(0) \int_{0}^{\infty} f(a) d a,
$$

where $f(a)$ is defined as follows:

$$
f(a)=k_{2}(a) \pi_{2}(a) .
$$

In the following proposition, we show that if $\lambda^{*}=0$, then the steady state of problem (2.3) is either the disease-free equilibrium or the trivial equilibrium.

Proposition 4.1. Suppose that $\lambda^{*}=0$. Then the steady is either the trivial equilibrium $s^{*}(a)=0, i^{*}(a)=0$, or the disease-free equilibrium

$$
s^{*}(a)=B^{*} \pi(a), \quad i^{*}(a)=0, \quad R_{0}=1 .
$$

Proof. If $\lambda^{*}=0$, then from (4.13) and assumption (2) in Section 2, $i^{*}(0)=0$; and from (4.9), $i^{*}(a)=0$. Therefore, from (4.1) and (4.8), $s^{*}(a)=B^{*} \pi(a)$.

From (4.1), we find that $B^{*}\left[1-R_{0}\right]=0$; and so if $R_{0}=1$ and $B^{*} \neq 0$, we obtain the disease-free equilibrium (4.15); otherwise, we obtain $B^{*}=0$, and accordingly, from (4.1) and (4.8), we obtain the trivial equilibrium $s^{*}(a)=0, i^{*}(a)=0$. This completes the proof of the proposition.

We note that it is easy to see that (4.15) solves problem (2.3).

We notice that from (4.11) and (4.12), and for a nontrivial equilibrium $\left(B^{*} \neq 0\right), T=0$ is equivalent to the following equations:

$$
\begin{gathered}
q R(\alpha)=1, \\
q \lambda^{*} \int_{0}^{\infty} \int_{0}^{a} h(a) F_{k_{1}}(\sigma) d \sigma=0 .
\end{gathered}
$$

In the following proposition, we show that in the special case $T=0$ and $R_{0}=1$, the steady state of problem (2.3) gives rise to a continuum of endemic equilibriums.

Proposition 4.2. Suppose that the following two conditions hold: (1) $R_{0}=q=1$, (2) the support of both $k_{1}(a)$ and $\alpha(a)$ lie to right of the support of $\beta(a)$. Then problem (2.3) gives rise to a continuum of endemic equilibriums.

Proof. From assumptions (1) and (2), we obtain that $R(\alpha)=1$, and therefore, (4.16) is satisfied; also by assumption (2), (4.17) is satisfied, and therefore, $T=0$. From (4.11), 
we see that $i^{*}(0)$ is undetermined. Using (4.9), (4.1), and (4.17), we obtain that $s^{*}(0)$ is also undetermined. Using (4.13), we see that for a fixed $i^{*}(0) \in\left(0, B^{*}\right]$ and a fixed $s^{*}(0) \in\left[0, B^{*}\right)$, the left-hand side of $(4.13)$ is an increasing function of $\lambda^{*}$ and approaches $+\infty$ as $\lambda^{*} \rightarrow \infty$, and equals zero if $\lambda^{*}=0$. Accordingly, for each $i^{*}(0) \in\left(0, B^{*}\right]$ and $s^{*}(0) \in\left[0, B^{*}\right)$, we obtain $\lambda^{*}>0$ as a solution of (4.13) which gives rise to an endemic equilibrium. We note that if $i^{*}(0)=0$, then from (4.13), either $\lambda^{*}=0$ and thus, the steady state is given by Proposition 4.1, or $\lambda^{*}>0$; and the latter exists if and only if the following condition holds: $s^{*}(0) \int_{0}^{\infty} \int_{0}^{a} f(a) J_{k_{1}}(\sigma) d \sigma d a>1$, where $J_{k_{1}}(\sigma)$ is defined as follows:

$$
J_{v}(\sigma)=v(\sigma) \exp \left(\int_{0}^{\sigma} \alpha(\tau) d \tau\right)
$$

This completes the proof of the proposition.

We note that Proposition 4.2 proves that the SI model of this paper and the SIR model studied in [8] behave in a similar fashion in this special case.

In the following proposition, we determine the steady state of problem (2.3) in the special case $T=0$ and $R_{0}>1$.

Proposition 4.3. Suppose that the following three conditions hold: (1) $R_{0}>1$, (2) $q R(\alpha)=$ 1 , and (3) the support of $k_{1}(a)$ lies to the right of the support of $\beta(a)$. Then the steady state of problem (2.3) is either the trivial equilibrium or is given by

$$
i^{*}(a)=B^{*} \pi_{2}(a), \quad s^{*}(a)=0, \quad R(\alpha)=1=q .
$$

Proof. From $B^{*}=\int_{0}^{\infty} \beta(a)\left[s^{*}(a)+i^{*}(a)\right] d a$ and (4.8), (4.9), and assumption (3), we obtain that

$$
B^{*}\left[R_{0}-1\right]=i^{*}(0)\left[R_{0}-R(\alpha)\right]
$$

So, if $R(\alpha) \neq R_{0}$, then we obtain the following:

$$
i^{*}(0)=\frac{B^{*}\left[R_{0}-1\right]}{\left[R_{0}-R(\alpha)\right]} .
$$

Using (4.1), we obtain that $s^{*}(0)$ satisfies

$$
s^{*}(0)=\frac{B^{*}[1-R(\alpha)]}{\left[R_{0}-R(\alpha)\right]} .
$$

From (4.22), we deduce that $R(\alpha) \leq 1$ since $s^{*}(0) \geq 0$, and hence $R(\alpha)=1=q$ by assumption (2). Accordingly, $s^{*}(0)=0$ and thus, $i^{*}(0)=B^{*}$ from (4.21); and hence we obtain (4.19) from (4.9).

Now, if $R(\alpha)=R_{0}$, then from (4.20), we obtain that $B^{*}=0$ since $R_{0}>1$; and hence the only steady state, in this case, is the trivial equilibrium $s^{*}(a)=i^{*}(a)=0$. This completes the proof of the proposition.

We note that it is easy to see that (4.19) solves problem (2.3). 
In the next proposition, we determine the steady state of problem (2.3) in the special case $T \neq 0$ and $q R(\alpha)=1$.

Proposition 4.4. Suppose that $q R(\alpha)=1$ and (4.17) does not hold. Then the steady state of problem (2.3) is either the trivial solution or is given by (4.19).

Proof. Since (4.17) does not hold, then $T \neq 0$ and thus, we can use (4.11) to obtain that $i^{*}(0)=B^{*}$; which implies that $s^{*}(0)=0$ and hence $s^{*}(a)=0$. By using (4.9), we obtain that $i^{*}(a)=B^{*} \pi_{2}(a)$. From (4.1), we obtain that $0=s^{*}(0)=(1-q) B^{*} R(\alpha)=(R(\alpha)-$ 1) $B^{*}$. Accordingly, either $R(\alpha)=1$, and therefore, $q=1$; and thus, we obtain (4.19) or $B^{*}=0$; and thus, the steady state is the trivial equilibrium. This completes the proof of the proposition.

Now, we consider the case $T \neq 0$ and therefore, we can use (4.11) to obtain that $i^{*}(0)$ satisfies

$$
i^{*}(0)=\frac{q \lambda^{*} B^{*}}{T} \int_{0}^{\infty} \int_{0}^{a} h(a) F_{k_{1}}(\sigma) d \sigma d a
$$

Using (4.23) and (4.1), we obtain that $s^{*}(0)$ satisfies

$$
s^{*}(0)=\frac{B^{*}}{T}[1-q R(\alpha)]
$$

Thus, using (4.23), (4.24), (4.9), (4.8), and (4.3), we obtain the following:

$\lambda^{*}\left[1-\frac{B^{*}}{T}\left\{\left(q \int_{0}^{\infty} f(a) d a\right) \int_{0}^{\infty} \int_{0}^{a} h(a) F_{k_{1}}(\sigma) d \sigma d a-(1-q R(\alpha)) \int_{0}^{\infty} \int_{0}^{a} f(a) F_{k_{1}}(\sigma) d \sigma d a\right\}\right]=0$.

In the following theorem, we describe the steady state of problem (2.3) in the case $R(\alpha)<1<R_{0}$. We note that $R_{0}>1$ describes a situation in which the population size would grow unboundedly, provided the disease is absent, and the theorem describes a situation in which the presence of the disease keeps the population size bounded by mortality.

Theorem 4.5. (1) Suppose that $R(\alpha)<1<R_{0}$. Then the steady state of problem (2.3) is either the trivial equilibrium, or is given by $\lambda^{*}>0$, which is the unique solution of the following characteristic equation:

$$
\begin{aligned}
1= & R(\alpha)+q[1-R(\alpha)] \int_{0}^{\infty} \beta(a) \pi(a) \exp \left(-\lambda^{*} \int_{0}^{a} k_{1}(\tau) d \tau\right) d a \\
& +(1-q) \int_{0}^{\infty} \int_{0}^{a} h(a) F_{\alpha}(\sigma) d \sigma d a .
\end{aligned}
$$

(2) Suppose that $R_{0}<1$. Then the trivial equilibrium $s^{*}(a)=i^{*}(a)=0$ is the only steady state. 
Proof. To prove (1), note that since $R(\alpha)<1$, then by $(4.12), T>0$. Therefore, using $B^{*}=$ $\int_{0}^{\infty} \beta(a)\left[s^{*}(a)+i^{*}(a)\right] d a$, and (4.8), (4.9), (4.23), and (4.24), we obtain that

$$
\begin{gathered}
B^{*}\left\{1-R(\alpha)-q[1-R(\alpha)] \int_{0}^{\infty} \beta(a) \pi(a) \exp \left(-\lambda^{*} \int_{0}^{a} k_{1}(\tau) d \tau\right) d a\right. \\
\left.-(1-q) \int_{0}^{\infty} \int_{0}^{a} h(a) F_{\alpha}(\sigma) d \sigma d a\right\}=0 .
\end{gathered}
$$

Accordingly, if the steady state is not trivial $\left(B^{*} \neq 0\right)$, then $\lambda^{*}$ satisfies (4.26).

Now, if the support of $k_{1}(a)$ lies to the right of the support of $\beta(a)$, that is, $\int_{0}^{\infty} \beta(a) \pi(a) \exp \left(-\lambda^{*} \int_{0}^{a} k_{1}(\tau) d \tau\right) d a=R_{0}$, then from (4.26), we obtain that $\left(R_{0}-1\right)[1-$ $q R(\alpha)]=0$, which is not possible since $R_{0}>1$ and $R(\alpha)<1$ by assumption (1). Therefore, (4.27) gives $B^{*}=0$; and therefore, in the case of nonfertile infectibles, the only steady state is the trivial equilibrium. If this special case does not occur, then we observe that the right-hand side of (4.26) is a decreasing function of $\lambda^{*}$ and has a value equal to $[1-q R(\alpha)] R_{0}+q R(\alpha)>1$, if $\lambda^{*}=0$, and tends to $R(\alpha)<1$ as $\lambda^{*} \rightarrow \infty$. Therefore, there exists a unique $\lambda^{*}>0$ which solves (4.26) and gives rise to a unique endemic equilibrium via (4.25), (4.12), (4.9) (4.23), (4.24), and (4.8). This proves (1).

On the other hand, if $R_{0}<1$, then this implies that $R(\alpha)<1$, and therefore, from (4.12), $T>0$. Thus, (4.27) holds; and accordingly as before, if the support of $k_{1}(a)$ lies to the right of the support of $\beta(a)$, then similar arguments to that given in the proof of (1) show that the only steady state, in this special case, is the trivial equilibrium. If this special case does not occur, then as before, the right-hand side of (4.26) is a decreasing function of $\lambda^{*}$, assumes the value $[1-q R(\alpha)] R_{0}+q R(\alpha)<1$, if $\lambda^{*}=0$, and tends to $R(\alpha)<1$ as $\lambda^{*} \rightarrow \infty$. Therefore, (4.26) and (4.27) give $B^{*}=0$, and thus, the only steady state for problem (2.3), if $R_{0}<1$, is the trivial equilibrium. This completes the proof of (2) and therefore, the proof of Theorem 4.5 is completed.

In the following proposition, we determine the steady state of problem (2.3) in the special case of nonfertile infectibles and $q R(\alpha)<1$.

Proposition 4.6. Suppose that $q R(\alpha)<1$ and the support of $k_{1}(a)$ lie to the right of the support of $\beta(a)$. Then the steady state of problem (2.3) is the trivial equilibrium if $R_{0} \neq 1$; and if $R_{0}=1$, then the steady state is either the one given by Proposition 4.1 or a continuum of endemic equilibriums.

Proof. From (4.9) and (4.2), we obtain $i^{*}(0)[1-q R(\alpha)]=0$ and thus, $i^{*}(0)=0$ since $q R(\alpha)<1$. Hence from (4.9) and (4.13), we obtain the following:

$$
\begin{gathered}
i^{*}(a)=\lambda^{*} s^{*}(0) \pi_{2}(a) \int_{0}^{a} F_{k_{1}}(\sigma) d \sigma, \\
\lambda^{*}\left[1-s^{*}(0) \int_{0}^{\infty} \int_{0}^{a} f(a) F_{k_{1}}(\sigma) d \sigma d a\right]=0 .
\end{gathered}
$$

Now, from (4.8), (4.1), and the assumption about the support of $k_{1}(a)$, we obtain that

$$
s^{*}(0)\left[1-R_{0}\right]=0 .
$$


So, if $R_{0} \neq 1$, then from (4.30), we deduce that $s^{*}(0)=0$, and hence (4.28) and (4.8) give the trivial equilibrium. Otherwise, if $R_{0}=1$, then (4.30) gives that $s^{*}(0)$ is undetermined. Accordingly, from (4.29), we see that either $\lambda^{*}=0$, and therefore, Proposition 4.1 gives the steady state, or

$$
1=s^{*}(0) \int_{0}^{\infty} \int_{0}^{a} f(a) F_{k_{1}}(\sigma) d \sigma d a
$$

We notice that the right-hand side of (4.31) is a decreasing function of $\lambda^{*}$ with a value greater than one (provided we choose $s^{*}(0)>\left[\int_{0}^{\infty} \int_{0}^{a} f(a) J_{k_{1}}(\sigma) d \sigma d a\right]^{-1}$ ) when $\lambda^{*}=0$ and approaches zero as $\lambda^{*} \rightarrow \infty$. Therefore, there exists $\lambda^{*}>0$ as a solution of (4.29) which gives rise to an endemic equilibrium. Thus, we obtain a continuum of endemic equilibriums. This completes the proof of the proposition.

In the following lemma, we prove that if $q R(\alpha)>1$, then the only steady state of problem (2.3) is the trivial equilibrium. Note that if $q=0$, that is, the case of no vertical transmission, then from (4.26), we can see that if $R(\alpha)>1$, then the only steady state of problem (2.3), in this special case, is the trivial equilibrium.

Lemma 4.7. Suppose that $q R(\alpha)>1$. Then the only steady state of problem (2.3) is the trivial equilibrium.

Proof. We note that from (4.9) and (4.2), we obtain that

$$
i^{*}(0)[1-q R(\alpha)]=q \lambda^{*} s^{*}(0) \int_{0}^{\infty} \int_{0}^{a} h(a) F_{k_{1}}(\sigma) d \sigma d a .
$$

Thus, (4.32) gives that $i^{*}(0)=0$ since $i^{*}(0) \geq 0$ and $q R(\alpha)>1$. Also from (4.32), we obtain that

$$
q \lambda^{*} B^{*} \int_{0}^{\infty} \int_{0}^{a} h(a) F_{k_{1}}(\sigma) d \sigma d a=0
$$

Now, from (4.8), (4.9), (4.33), and (4.1), we obtain that

$$
B^{*}\left[1-\int_{0}^{\infty} \beta(a) \pi(a) \exp \left(-\lambda^{*} \int_{0}^{a} k_{1}(\tau) d \tau\right) d a\right]=0 .
$$

Therefore, from (4.34), we see that either the steady state is trivial $\left(B^{*}=0\right)$ or

$$
1=\int_{0}^{\infty} \beta(a) \pi(a) \exp \left(-\lambda^{*} \int_{0}^{a} k_{1}(\tau) d \tau\right) d a
$$

By integrating (4.12) by parts and using (4.35), we conclude that $T \geq 0$. Accordingly, (4.33) gives the result since $B^{*}$ must be equal to zero; otherwise, $T$ is negative. Note that $T>0$ gives $s^{*}(0)=0$ by (4.24) since $s^{*}(0) \geq 0$. This completes the proof of the lemma. 
In the following theorem, we prove that problem (2.3) has an endemic equilibrium, when $q R(\alpha)<1<R_{0}$. Note that Lemma 4.7 proved that if $q R(\alpha)>1$, then the only steady state for problem (2.3) is the trivial equilibrium.

Theorem 4.8. Suppose that $q R(\alpha)<1<R_{0}$. Then the steady state of problem (2.3) is either the trivial equilibrium or is given by $\lambda^{*}>0$ which is a solution of the following characteristic equation:

$$
\begin{aligned}
1= & q R(\alpha)+[1-q R(\alpha)] \int_{0}^{\infty} \beta(a) \pi(a) \exp \left(-\lambda^{*} \int_{0}^{a} k_{1}(\tau) d \tau\right) d a \\
& +\lambda^{*}(1-q) \int_{0}^{\infty} \int_{0}^{a} h(a) F_{k_{1}}(\sigma) d \sigma d a .
\end{aligned}
$$

Proof. We note that $T>0$ since $q R(\alpha)<1$, and therefore, using (4.26), we obtain (4.36) by integration by parts.

Now, if the support of $k_{1}(a)$ lies to the right of the support of $\beta(a)$, then similar arguments to that given in the proof of Theorem 4.5 show that the only steady state, in this special case, is the trivial equilibrium. If this special case does not occur; then the right-hand side of (4.36) assumes the value $[1-q R(\alpha)] R_{0}+q R(\alpha)>1$, if $\lambda^{*}=0$, and approaches $q R(\alpha)<1$ as $\lambda^{*} \rightarrow \infty$. Therefore, (4.36) has a solution $\lambda^{*}>0$, which gives rise to an endemic equilibrium via (4.23), (4.24), (4.12), (4.9), and (4.8). This completes the proof of the theorem.

We note that this endemic equilibrium may not be unique since the right-hand side of (4.36) may not be monotone.

\section{Stability of the steady states}

In this section, we study the stability of the steady states for problem (2.3) as given by the results in Section 4. Note that as in Section 4, we will continue to define $F_{v}, f, h$, and $J_{v}$, respectively, by (4.10), (4.14), (4.5), and (4.18) throughout this section.

By integrating problem (3.3) along characteristics lines $t-a=$ constant, we find that

$$
s(a, t)= \begin{cases}s_{0}(a-t) e^{-\int_{0}^{t}\left[\mu(a-t+\tau)+k_{1}(a-t+\tau) \lambda(\tau)\right] d \tau}, & a>t, \\ {[B(t-a)-i(0, t-a)] \pi(a) e^{-\int_{0}^{a} k_{1}(\tau) \lambda(t-a+\tau) d \tau},} & a<t .\end{cases}
$$

By integrating problem (3.2) along characteristics lines $t-a=$ constant, we find that

$$
\begin{aligned}
& p(a, t) \\
& = \begin{cases}p_{0}(a-t) \exp \left(-\int_{0}^{t}[\mu(a-t+\tau)+\alpha(a-t+\tau)] d \tau\right) \\
\quad+\int_{0}^{t} \exp \left(-\int_{\sigma}^{t}[\mu(a-t+\tau)+\alpha(a-t+\tau)] d \tau\right) \alpha(a-t+\sigma) s(a-t+\sigma, \sigma) d \sigma, & a>t, \\
B(t-a) \pi_{2}(a)+\int_{0}^{a} \exp \left(-\int_{\sigma}^{a}[\mu(\tau)+\alpha(\tau)] d \tau\right) \alpha(\sigma) s(\sigma, t-a+\sigma) d \sigma, & a<t .\end{cases}
\end{aligned}
$$


246 An age-structured SI epidemic model with fatalities

From (3.2) and (5.2), we find that

$$
\begin{aligned}
B(t)= & \int_{0}^{t} h(a) B(t-a) d a+\int_{0}^{t} \int_{0}^{\infty} \beta(a+\sigma) \exp \left(-\int_{a}^{a+\sigma}[\mu(\tau)+\alpha(\tau)] d \tau\right) \alpha(a) s(a, t-\sigma) d a d \sigma \\
& +\int_{t}^{\infty} \beta(a) \exp \left(-\int_{a}^{a+\sigma}[\mu(a-t+\tau)+\alpha(a-t+\tau)] d \tau\right) p_{0}(a-t) d a .
\end{aligned}
$$

By integrating problem (3.4) along characteristics lines $t-a=$ constant, we find that

$$
\begin{aligned}
& i(a, t) \\
& = \begin{cases}i_{0}(a-t) \exp \left(-\int_{0}^{t}[\mu(a-t+\tau)+\alpha(a-t+\tau)] d \tau\right) & \\
\quad+\int_{0}^{t} \exp \left(-\int_{\sigma}^{t}[\mu(a-t+\tau)+\alpha(a-t+\tau)] d \tau\right) k_{1}(a-t+\sigma) s(a-t+\sigma, \sigma) \lambda(\sigma) d \sigma, & a>t, \\
i(0, t-a) \pi_{2}(a)+\int_{0}^{a} \exp \left(-\int_{\sigma}^{a}[\mu(\tau)+\alpha(\tau)] d \tau\right) k_{1}(\sigma) s(\sigma, t-a+\sigma) \lambda(t-a+\sigma) d \sigma, & a<t .\end{cases}
\end{aligned}
$$

From (3.4) and (5.4), we find that

$$
\begin{aligned}
\lambda(t)= & \int_{0}^{t} \int_{0}^{\infty} k_{2}(a+\sigma) \exp \left(-\int_{a}^{a+\sigma}[\mu(\tau)+\alpha(\tau)] d \tau\right) k_{1}(a) s(a, t-\sigma) \lambda(t-\sigma) d a d \sigma \\
& +\int_{t}^{\infty} k_{2}(a) i_{0}(a-t) \exp -\int_{0}^{t}[\mu(a-t+\tau)+\alpha(a-t+\tau)] d a+\int_{0}^{t} f(a) i(0, t-a) d a .
\end{aligned}
$$

Setting $i(0)=V(t)$ and using (3.4) and (5.4), we obtain that

$$
\begin{aligned}
V(t)= & q \int_{0}^{t} h(a) V(t-a) d a \\
& +q \int_{0}^{t} \int_{0}^{\infty} \beta(a+\sigma) \exp \left(-\int_{a}^{a+\sigma}[\mu(\tau)+\alpha(\tau)] d \tau\right) k_{1}(a) s(a, t-\sigma) \lambda(t-\sigma) d a d \sigma \\
& +q \int_{t}^{\infty} \beta(a) i_{0}(a-t) \exp \left(-\int_{0}^{a}[\mu(a-t+\tau)+\alpha(a-t+\tau)] d \tau\right) d a .
\end{aligned}
$$

We note that, by assumptions (3), (4), and (6) in Section 2, and the dominated convergence theorem,

$$
\int_{t}^{\infty} \beta(a) p_{0}(a-t) \exp \left(-\int_{0}^{t}[\mu(a-t+\tau)+\alpha(a-t+\tau)] d \tau\right) d a \longrightarrow 0, \quad \text { as } t \longrightarrow \infty .
$$


Also, by the same reasoning as above,

$$
\begin{array}{ll}
\int_{t}^{\infty} k_{2}(a) i_{0}(a-t) \exp \left(-\int_{0}^{t}[\mu(a-t+\tau)+\alpha(a-t+\tau)] d \tau\right) d a \longrightarrow 0, & \text { as } t \longrightarrow \infty, \\
q \int_{t}^{\infty} \beta(a) i_{0}(a-t) \exp \left(-\int_{0}^{t}[\mu(a-t+\tau)+\alpha(a-t+\tau)] d \tau\right) d a \longrightarrow 0, & \text { as } t \longrightarrow \infty .
\end{array}
$$

Consequently, $B(t), \lambda(t)$, and $V(t)$ satisfy the following limiting equations (see Miller [20]):

$$
\begin{aligned}
& B(t)= \int_{0}^{\infty} h(a) B(t-a) d a \\
&+\int_{0}^{\infty} \int_{0}^{\infty} h(a+\sigma)[B(t-a-\sigma)-V(t-a-\sigma)] J_{\alpha}(a) \exp \left(-\int_{0}^{a} k_{1}(\tau) \lambda(t-a-\sigma+\tau) d \tau\right) d a d \sigma, \\
& \lambda(t)= \int_{0}^{\infty} f(a) V(t-a) d a+\int_{0}^{\infty} \int_{0}^{\infty} f(a+\sigma) J_{k_{1}}(a)[B(t-a-\sigma)-V(t-a-\sigma)] \\
& \times \exp \left(-\int_{0}^{a} k_{1}(\tau) \lambda(t-a+\sigma+\tau) d \tau\right) \lambda(t-\sigma) d a d \sigma, \\
& V(t)= q \int_{0}^{\infty} h(a) V(t-a) d a+q \int_{0}^{\infty} \int_{0}^{\infty} h(a+\sigma)[B(t-a-\sigma)-V(t-a-\sigma)] \\
& \times J_{k_{1}}(a) \exp \left(-\int_{0}^{a} k_{1}(\tau) \lambda(t-a-\sigma+\tau) d \tau\right) \lambda(t-\sigma) d a d \sigma .
\end{aligned}
$$

Now, we linearize the system of (5.9) by considering perturbations $w(t), \eta(t)$, and $\zeta(t)$ defined by

$$
w(t)=\lambda(t)-\lambda^{*}, \quad \eta(t)=B(t)-B^{*}, \quad \zeta(t)=V(t)-V^{*} .
$$

Now, if we define

$$
x(t)=\left(\begin{array}{c}
w(t) \\
\eta(t) \\
\zeta(t)
\end{array}\right),
$$

then the linearization of (5.9) can be rewritten as follows:

$$
x(t)=\int_{0}^{\infty} A(\sigma) x(t-\sigma) d \sigma
$$

where $A(\sigma)$ is given by

$$
A(\sigma)=\left[\begin{array}{ccc}
Y_{11} & \lambda^{*} f(\sigma) \int_{0}^{\sigma} F_{k_{1}}(a) d a & f(\sigma)\left[1-\lambda^{*} \int_{0}^{\sigma} F_{k_{1}}(a) d a\right] \\
Y_{12} & h(\sigma)\left[1+\int_{0}^{\sigma} F_{\alpha}(a) d a\right] & -h(\sigma) \int_{0}^{\sigma} F_{\alpha}(a) d a \\
Y_{13} & \lambda^{*} q h(\sigma) \int_{0}^{\sigma} F_{k_{1}}(a) d a & q h(\sigma)\left[1-\lambda^{*} \int_{0}^{\sigma} F_{k_{1}}(a) d a\right]
\end{array}\right],
$$


where

$$
\begin{aligned}
& Y_{11}=\left(B^{*}-V^{*}\right)\left[\int_{0}^{\infty} F_{k_{1}}(a) f(a+\sigma) d a-\lambda^{*} \int_{0}^{\sigma} \int_{0}^{\infty} f(a+\sigma) F_{k_{1}}(a+\tau) d a d \tau\right], \\
& Y_{12}=\left(V^{*}-B^{*}\right) \int_{0}^{\sigma} \int_{0}^{\infty} h(a+\sigma) F_{\alpha}(a+\tau) k_{1}(a) d a d \tau \\
& Y_{13}=q\left(B^{*}-V^{*}\right)\left[\int_{0}^{\infty} h(a+\sigma) F_{k_{1}}(a) d a-\lambda^{*} \int_{0}^{\sigma} \int_{0}^{\infty} h(a+\sigma) k_{1}(a) F_{k_{1}}(a+\tau) d a d \tau\right] .
\end{aligned}
$$

In the following theorem, we show that the trivial equilibrium $s^{*}(a)=i^{*}(a)=0$ is unstable if $R_{0}>1$ and locally asymptotically stable if $R_{0}<1$.

THeOrem 5.1. The trivial equilibrium $B^{*}=0$ is unstable if $R_{0}>1$ and locally asymptotically stable if $R_{0}<1$.

Proof. We note that the characteristic equation for the system (5.12) is given in Appendix A.

If $B^{*}=0$, then $\lambda^{*}=V^{*}=0$, whence the characteristic equation (A.1) becomes [ $1-$ $\left.q \int_{0}^{\infty} e^{-\sigma z} h(\sigma) d \sigma\right]\left\{1-\int_{0}^{\infty} e^{-\sigma z} h(\sigma) d \sigma-\int_{0}^{\infty} \int_{0}^{\sigma} e^{-\sigma z} h(\sigma) J_{\alpha}(a) d a d \sigma\right\}=0$, and therefore,

$$
\left[1-q \int_{0}^{\infty} e^{-\sigma z} h(\sigma) d \sigma\right]\left[1-\int_{0}^{\infty} e^{-\sigma z} \beta(\sigma) \pi(\sigma) d \sigma\right]=0
$$

Setting $z=x+i y$ and if we suppose that $1=\int_{0}^{\infty} e^{-\sigma(x+i y)} \beta(\sigma) \pi(\sigma) d \sigma$, then we obtain that

$$
\begin{aligned}
& 1=\int_{0}^{\infty} e^{-\sigma x} \beta(\sigma) \pi(\sigma) \cos \sigma y d \sigma, \\
& 0=\int_{0}^{\infty} e^{-\sigma x} \beta(\sigma) \pi(\sigma) \sin \sigma y d \sigma .
\end{aligned}
$$

If $R_{0}>1$, then from (5.16), we see that if we set $y=0$ and define a function $g(x)$ by

$$
g(x)=\int_{0}^{\infty} e^{-\sigma x} \beta(\sigma) \pi(\sigma) d \sigma
$$

then $g(x)$ is a decreasing function for $x>0, g(x) \rightarrow 0$ as $x \rightarrow \infty$, and $g(0)=R_{0}>1$. Therefore, there exists $x^{*}>0$ such that $g\left(x^{*}\right)=1$. Accordingly, the trivial equilibrium is unstable.

If $R_{0}<1$, then (5.16) cannot be satisfied for $x \geq 0$. Also, by similar argument, we see that $1-q \int_{0}^{\infty} e^{-\sigma z} h(\sigma) d \sigma=0$ cannot be satisfied for $x \geq 0$. Therefore, the trivial equilibrium is locally asymptotically stable if $R_{0}<1$. This completes the proof of the theorem. 
In the next theorem, we show that the trivial equilibrium $B^{*}=0$ is globally stable if $R_{0}<1$. A proof of this theorem is essentially contained in [5], and therefore, we omit the proof.

Theorem 5.2. Suppose that $R_{0}<1$. Then the trivial equilibrium is globally stable.

In the following theorem, we show that the steady state given by Proposition 4.3 is always unstable, whereas the steady state given by Proposition 4.4 is either locally asymptotically stable or unstable, depending on a threshold parameter.

Theorem 5.3. (1) The steady state given by Proposition 4.3 is unstable.

(2) The steady state given by Proposition 4.4 is locally asymptotically stable if $\int_{0}^{\infty} \beta(a) \pi(a) \exp \left(-\lambda^{*} \int_{0}^{a} k_{1}(c) d c\right) d a<1$, and unstable if $\int_{0}^{\infty} \beta(a) \pi(a) \exp \left(-\lambda^{*} \int_{0}^{a} k_{1}(c) d c\right) d a$ $>1$.

Proof. To prove (1), we note that in this case, $B^{*}=V^{*}$, and therefore, the characteristic equation (A.1) satisfies the following:

$$
\left[1-\int_{0}^{\infty} e^{-\sigma z} h(\sigma) d \sigma\right]\left[1-\int_{0}^{\infty} e^{-\sigma z} \beta(\sigma) \pi(\sigma) \exp \left(-\lambda^{*} \int_{0}^{\sigma} k_{1}(c) d c\right) d \sigma\right]=0 .
$$

Whence, by assumption (3) of Proposition 4.3, we obtain that

$$
\int_{0}^{\infty} \beta(a) \pi(a) \exp \left(-\lambda^{*} \int_{0}^{a} k_{1}(c) d c\right) d a=\int_{0}^{\infty} \beta(a) \pi(a) d a=R_{0}>1 .
$$

Therefore, this steady state is unstable by similar arguments as given in the proof of Theorem 5.1. This completes the proof of (1). The proof of (2) is similar and therefore is omitted. This completes the proof of the theorem.

We note that if we set $\lambda^{*}=0$, then the characteristic equation (A.1) satisfies the following:

$$
\begin{aligned}
{\left[1-\int_{0}^{\infty} e^{-a z} \beta(a) \pi(a) d a\right]\{} & {\left[1-q \int_{0}^{\infty} e^{-a z} h(a) d a\right] } \\
& \times\left[1-B^{*} \int_{0}^{\infty} \int_{0}^{\infty} e^{-\sigma z} f(a+\sigma) J_{k_{1}}(a) d a d \sigma\right] \\
& \left.-q B^{*}\left(\int_{0}^{\infty} e^{-a z} f(a) d a\right) \int_{0}^{\infty} \int_{0}^{\infty} e^{-\sigma z} h(a+\sigma) J_{k_{1}}(a) d a d \sigma\right\}=0 .
\end{aligned}
$$

Then if we set $B^{*}=0$, we see that we can recover the stability result for the trivial equilibrium given by Theorem 5.1; but in general, the stability of the disease-free equilibrium given by Proposition 4.1 is not determined due to lack of information about $B^{*}$.

Anticipating our future needs, we define a function $D\left(b ; \lambda^{*} ; B^{*}\right)$ in Appendix B.

In the following theorem, we show that in the special case $q=0$, the endemic equilibrium with $\lambda^{*}>0$ is locally asymptotically stable when $R(\alpha)<1<R_{0}$. 
Theorem 5.4. Suppose that

(1) $R(\alpha)<1<R_{0}$,

(2) $d D\left(b ; \lambda^{*} ; B^{*}\right) / d b \leq 0$,

(3) $\beta(0)+B^{*} \int_{0}^{\infty} f(a) F_{k_{1}}(a) d a>0$.

Then the unique endemic steady state with $\lambda^{*}>0$ is locally asymptotically stable.

Proof. We start by noting that the imaginary part of the characteristic equation (A.1) can be rewritten in the following form:

$$
\int_{0}^{\infty} e^{-x b} \sin y b D\left(b ; \lambda^{*} ; B^{*}\right) d b=0
$$

We also note that $D\left(0 ; \lambda^{*} ; B^{*}\right)=\beta(0)+B^{*} \int_{0}^{\infty} f(a) F_{k_{1}}(a) d a>0$ by assumption (3).

We can check that $D\left(b ; \lambda^{*} ; B^{*}\right) \rightarrow 0$ as $b \rightarrow \infty$ since $\beta(b)$ has compact support; and $\mu(a)$ is nonnegative and eventually positive and increasing. Therefore, by assumption (2), $D\left(b ; \lambda^{*} ; B^{*}\right)$ decreases to zero as $b \rightarrow \infty$ and $e^{-x b} D\left(b ; \lambda^{*} ; B^{*}\right)$ decreases to zero for $x \geq 0$. Accordingly,

$$
\int_{0}^{\infty} e^{-x b} \sin y b D\left(b ; \lambda^{*} ; B^{*}\right) d b \neq 0, \quad \text { if } y \neq 0 .
$$

If $y=0$, then the real part of the characteristic equation (A.1) satisfies

$$
\begin{aligned}
& {\left[1-B^{*} \int_{0}^{\infty} \int_{0}^{\infty} e^{-x \sigma} f(a+\sigma) F_{k_{1}}(a) d a d \sigma\right.} \\
& \left.+\lambda^{*} B^{*} \int_{0}^{\infty} \int_{0}^{\sigma} \int_{0}^{\infty} e^{-x \sigma} f(a+\sigma) F_{k_{1}}(a+\tau) k_{1}(a) d a d \tau d \sigma\right] X \\
& {\left[1-\int_{0}^{\infty} e^{-x \sigma} h(\sigma) d \sigma-\int_{0}^{\infty} \int_{0}^{\sigma} e^{-x \sigma} h(\sigma) F_{\alpha}(a) d a d \sigma\right]} \\
& \quad+\lambda^{*} B^{*}\left[\int_{0}^{\infty} \int_{0}^{\sigma} e^{-x \sigma} f(\sigma) F_{k_{1}}(a) d a d \sigma\right] \\
& \quad \times\left[\int_{0}^{\infty} \int_{0}^{\sigma} \int_{0}^{\infty} e^{-x \sigma} h(a+\sigma) F_{\alpha}(a+\tau) k_{1}(a) d a d \tau d \sigma\right]=0 .
\end{aligned}
$$

Now, using (4.26) and (4.25), we get that (5.24) cannot be satisfied when $x \geq 0$. Accordingly, the steady state with $\lambda^{*}>0$ is locally asymptotically stable. This completes the proof of the theorem.

In the general case $q \neq 0$, we can show that the endemic equilibrium is locally asymptotically stable as in Theorem 5.4. But observe that the function $D\left(b ; \lambda^{*} ; B^{*} ; V^{*}\right)$, which corresponds to the function $D\left(b ; \lambda^{*} ; B^{*}\right)$, in the special case $q=0$, takes several pages to write, and therefore, we omit the details of the proof. 


\section{Appendices}

\section{A. The characteristic equation for the system (5.12)}

$$
\begin{aligned}
& \left\{1-\left(B^{*}-V^{*}\right)\left[\int_{0}^{\infty} \int_{0}^{\infty} e^{-\sigma z} f(a+\sigma) F_{k_{1}}(a) d a d \sigma\right.\right. \\
& \left.\left.-\lambda^{*} \int_{0}^{\infty} \int_{0}^{\sigma} \int_{0}^{\infty} e^{-\sigma z} f(a+\sigma) F_{k_{1}}(a+\tau) k_{1}(a) d a d \tau d \sigma\right]\right\} \\
& \times\left\{\left[1-\int_{0}^{\infty} e^{-\sigma z} h(\sigma) d \sigma-\int_{0}^{\infty} \int_{0}^{\sigma} e^{-\sigma z} h(\sigma) F_{\alpha}(a) d a d \sigma\right]\right. \\
& \times\left[1-q \int_{0}^{\infty} e^{-\sigma z} h(\sigma) d \sigma+q \lambda^{*} \int_{0}^{\infty} \int_{0}^{\sigma} e^{-\sigma z} h(\sigma) F_{k_{1}}(a) d a d \sigma\right] \\
& \left.+\lambda^{*} q\left(\int_{0}^{\infty} \int_{0}^{\sigma} e^{-\sigma z} h(\sigma) F_{\alpha}(a) d a d \sigma\right)\left(\int_{0}^{\infty} \int_{0}^{\sigma} e^{-\sigma z} h(\sigma) F_{k_{1}}(a) d a d \sigma\right)\right\} \\
& +\lambda^{*} \int_{0}^{\infty} \int_{0}^{\sigma} e^{-\sigma z} f(\sigma) F_{k_{1}}(a) d a d \sigma \\
& \times\left\{\left(B^{*}-V^{*}\right) \int_{0}^{\infty} \int_{0}^{\sigma} \int_{0}^{\infty} e^{-\sigma z} h(a+\sigma) F_{\alpha}(a+\tau) k_{1}(a) d a d \tau d \sigma\right. \\
& \times\left[1-q \int_{0}^{\infty} e^{-\sigma z} h(\sigma) d \sigma+q \lambda^{*} \int_{0}^{\infty} \int_{0}^{\sigma} e^{-\sigma z} h(\sigma) F_{k_{1}}(a) d a d \sigma\right] \\
& +q\left(B^{*}-V^{*}\right) \int_{0}^{\infty} \int_{0}^{\sigma} e^{-\sigma z} h(\sigma) F_{\alpha}(a) d a d \sigma \\
& \times\left[\int_{0}^{\infty} \int_{0}^{\infty} e^{-\sigma z} h(a+\sigma) F_{k_{1}}(a) d a d \sigma\right. \\
& \left.\left.-\lambda^{*} \int_{0}^{\infty} \int_{0}^{\sigma} \int_{0}^{\infty} e^{-\sigma z} h(a+\sigma) F_{k_{1}}(a+\tau) k_{1}(a) d a d \tau d \sigma\right]\right\} \\
& +\left[\lambda^{*} \int_{0}^{\infty} \int_{0}^{\sigma} e^{-\sigma z} f(\sigma) F_{k_{1}}(a) d a d \sigma-\int_{0}^{\infty} e^{-\sigma z} f(\sigma) d \sigma\right] \\
& \times\left\{\lambda^{*} q\left(V^{*}-B^{*}\right) \int_{0}^{\infty} \int_{0}^{\sigma} \int_{0}^{\infty} e^{-\sigma z} h(a+\sigma) F_{\alpha}(a+\tau) k_{1}(a) d a d \tau d \sigma\right. \\
& \times\left(\int_{0}^{\infty} \int_{0}^{\sigma} e^{-\sigma z} h(\sigma) F_{k_{1}}(a) d a d \sigma\right) \\
& +q\left(B^{*}-V^{*}\right)\left[1-\int_{0}^{\infty} e^{-\sigma z} h(\sigma) d \sigma-\int_{0}^{\infty} \int_{0}^{\sigma} e^{-\sigma z} h(\sigma) F_{\alpha}(a) d a d \sigma\right] \\
& \times\left[\int_{0}^{\infty} \int_{0}^{\infty} e^{-\sigma z} h(a+\sigma) F_{k_{1}}(a) d a d \sigma\right. \\
& \left.\left.-\lambda^{*} \int_{0}^{\infty} \int_{0}^{\sigma} \int_{0}^{\infty} e^{-\sigma z} h(a+\sigma) F_{k_{1}}(a+\tau) k_{1}(a) d a d \tau d \sigma\right]\right\}=0,
\end{aligned}
$$

where $F_{v}(\sigma), f(a)$, and $h(a)$ are given, respectively, by (4.10), (4.14), and (4.5). 
B. The function $D\left(b ; \lambda^{*} ; B^{*}\right)$

$$
\begin{aligned}
& D\left(b ; \lambda^{*} ; B^{*}\right)=h(b)+h(b) \int_{0}^{b} F_{\alpha}(a) d a \\
& +B^{*}\left\{\int_{0}^{\infty} f(a+b) F_{k_{1}}(a) d a\right. \\
& -\int_{0}^{\infty} \int_{0}^{b} \int_{0}^{b} h(b-\sigma) f(a+\sigma) F_{k_{1}}(a) F_{\alpha}(m) d m d \sigma d a \\
& \left.-\int_{0}^{\infty} \int_{0}^{b} h(b-\sigma) f(a+\sigma) F_{k_{1}}(a) d \sigma d a\right\} \\
& +\lambda^{*} B^{*}\left\{\int_{0}^{\infty} \int_{0}^{b} \int_{0}^{b} \int_{0}^{\sigma} f(a+\sigma) F_{k_{1}}(a+\tau) k_{1}(a) F_{\alpha}(m) h(b-\sigma) d \tau d m d \sigma d a\right. \\
& -\int_{0}^{\infty} \int_{0}^{b} \int_{0}^{b} \int_{0}^{\sigma} f(b-\sigma) h(a+\sigma) F_{\alpha}(a+\tau) k_{1}(a) F_{k_{1}}(m) d \tau d m d \sigma d a \\
& +\int_{0}^{b} \int_{0}^{\infty} \int_{0}^{\sigma} h(b-\sigma) f(a+\sigma) F_{k_{1}}(a+\tau) k_{1}(a) d \tau d a d \sigma \\
& \left.-\int_{0}^{b} \int_{0}^{\infty} f(a+b) F_{k_{1}}(a+\tau) k_{1}(a) d a d \tau\right\} \text {, }
\end{aligned}
$$

where $F_{v}(\sigma), f(a)$, and $h(a)$ are given, respectively, by (4.10), (4.14), and (4.5).

\section{Acknowledgments}

This paper was completed while the author was visiting The Abdus Salam ICTP, and he wishes to thank the Director Professor M. A. Virasoro for invitation and hospitality at the centre during his stay. The author would like to thank Professor Mimmo Iannelli, Professor Herbert W. Hethcote, Professor Horst R. Thieme, Professor G. C. Wake, Professor K. Dietz, Professor Michel Langlais, and Professor Carlos Castillo-Chavez for giving him references. This research was supported in part by the Third World Academy of Sciences (TWAS) under Research Grant Agreement no. 98-364 RG/MATHS/AF/AC.

\section{References}

[1] R. M. Anderson and R. M. May, Infectious Diseases of Humans: Dynamics and Control, Oxford University Press, Oxford, 1991.

[2] Understanding the AIDS pandemic, Scientific American 266 (1992), no. 5, 58-66.

[3] R. M. Anderson, R. M. May, and A. R. McLean, Possible demographic consequences of AIDS in developing countries, Nature 322 (1988), no. 6161, 228-234.

[4] V. Andreasen, Disease regulation of age-structured host populations, Theoret. Population Biol. 36 (1989), no. 2, 214-239.

[5] S. Busenberg and K. Cooke, Vertically Transmitted Diseases. Models and Dynamics, Biomathematics, vol. 23, Springer-Verlag, Berlin, 1993.

[6] S. Busenberg and K. L. Cooke, The population dynamics of two vertically transmitted infections, Theoret. Population Biol. 33 (1988), no. 2, 181-198. 
[7] S. N. Busenberg and K. P. Hadeler, Demography and epidemics, Math. Biosci. 101 (1990), no. 1, 63-74.

[8] Y. Cha, M. Iannelli, and F. A. Milner, Existence and uniqueness of endemic states for the agestructured S-I-R epidemic model, Math. Biosci. 150 (1998), no. 2, 177-190.

[9] K. Dietz and D. Schenzle, Proportionate mixing models for age-dependent infection transmission, J. Math. Biol. 22 (1985), no. 1, 117-120.

[10] M. El-Doma, Analysis of nonlinear integro-differential equations arising in age-dependent epidemic models, Nonlinear Anal. TMA 11 (1987), no. 8, 913-937.

[11] - Analysis of a general age-dependent vaccination model for a vertically transmitted disease, Nonlinear Times and Digest 2 (1995), no. 2, 147-171.

[12] _ Analysis of an age-dependent SI epidemic model with disease-induced mortality and proportionate mixing assumption, Int. J. Appl. Math. 3 (2000), no. 3, 233-247.

[13] Stability analysis of a general age-dependent vaccination model for a vertically transmitted disease under the proportionate mixing assumption, IMA J. Math. Appl. Med. Biol. 17 (2000), no. 2, 119-136.

[14] P. E. Fine, Vectors and vertical transmissions: an epidemiologic perspective, Ann. N. Y. Acad. Sci. 266 (1975), 173-194.

[15] H. W. Hethcote and J. W. Van Ark, Epidemiological models for heterogeneous populations: proportionate mixing, parameter estimation, and immunization programs, Math. Biosci. 84 (1987), no. $1,85-118$.

[16] W. M. Liu, H. W. Hethcote, and S. A. Levin, Dynamical behavior of epidemiological models with nonlinear incidence rates, J. Math. Biol. 25 (1987), no. 4, 359-380.

[17] K. Louie, M. G. Roberts, and G. C. Wake, The regulation of an age-structured population by a fatal disease, IMA J. Math. Appl. Med. Biol. 11 (1994), no. 4, 229-244.

[18] R. M. May, R. M. Anderson, and A. R. McLean, Possible demographic consequences of HIV/AIDS epidemics. I. Assuming HIV infection always leads to AIDS, Math. Biosci. 90 (1988), no. 1-2, 475-505.

[19] _ Possible demographic consequences of HIV/AIDS epidemics. II. Assuming HIV infection does not necessarily lead to AIDS, Mathematical Approaches to Problems in Resource Management and Epidemiology (New York, 1987) (C. Castillo-Chavez, S. A. Levin, and C. A. Shoemaker, eds.), Lecture Notes in Biomath., vol. 81, Springer, Berlin, 1989, pp. 220-248.

[20] R. K. Miller, Nonlinear Volterra Integral Equations, W. A. Benjamin, California, 1971.

[21] A. Pugliese, Population models for diseases with no recovery, J. Math. Biol. 28 (1990), no. 1, $65-82$.

[22] H. R. Thieme, Epidemic and demographic interaction in the spread of potentially fatal diseases in growing populations, Math. Biosci. 111 (1992), no. 1, 99-130.

M. El-Doma: Faculty of Mathematical Sciences, University of Khartoum, P.O. Box 321, 11115 Khartoum, Sudan

E-mail address: meldoma@yahoo.com 


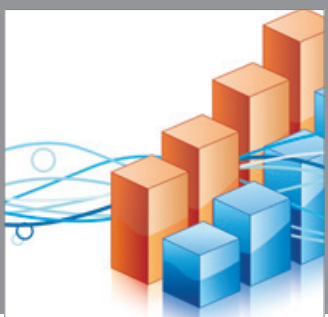

Advances in

Operations Research

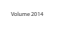

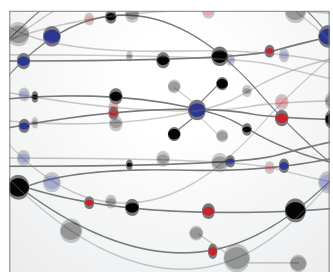

\section{The Scientific} World Journal
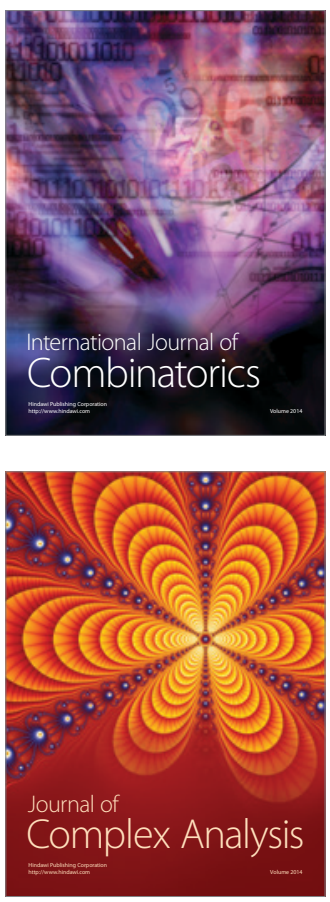

International Journal of

Mathematics and

Mathematical

Sciences
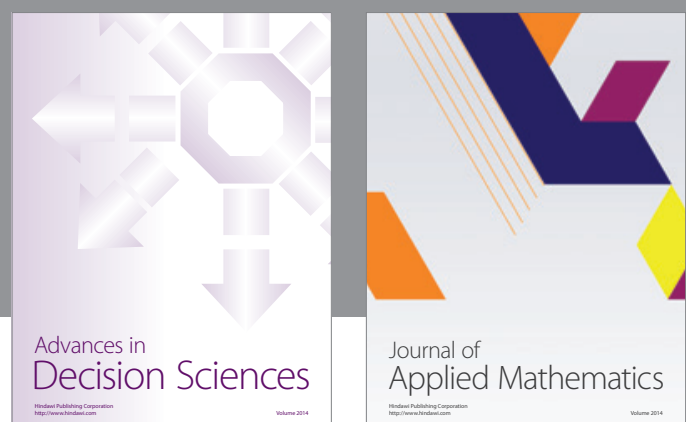

Journal of

Applied Mathematics
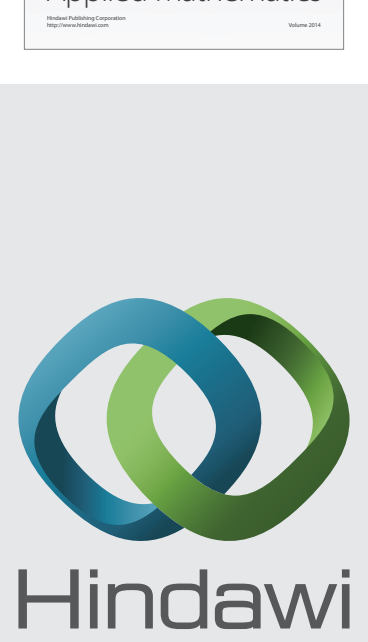

Submit your manuscripts at http://www.hindawi.com
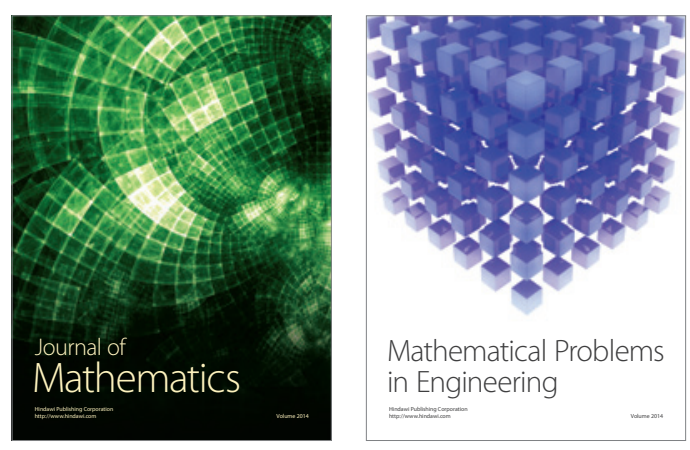

Mathematical Problems in Engineering
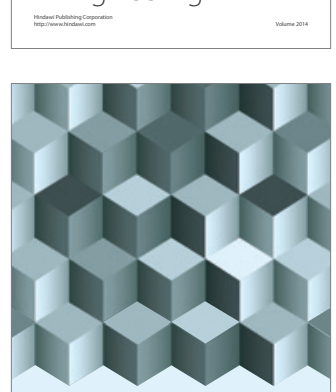

Journal of

Function Spaces
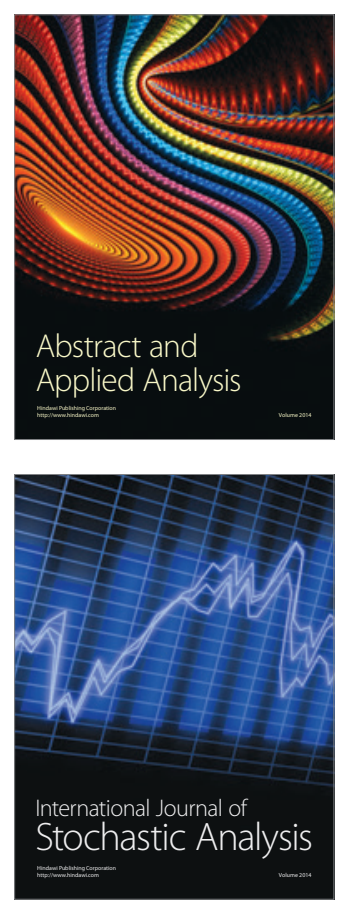

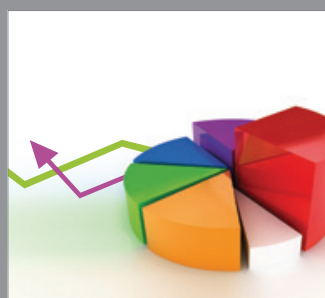

ournal of

Probability and Statistics

Promensencen
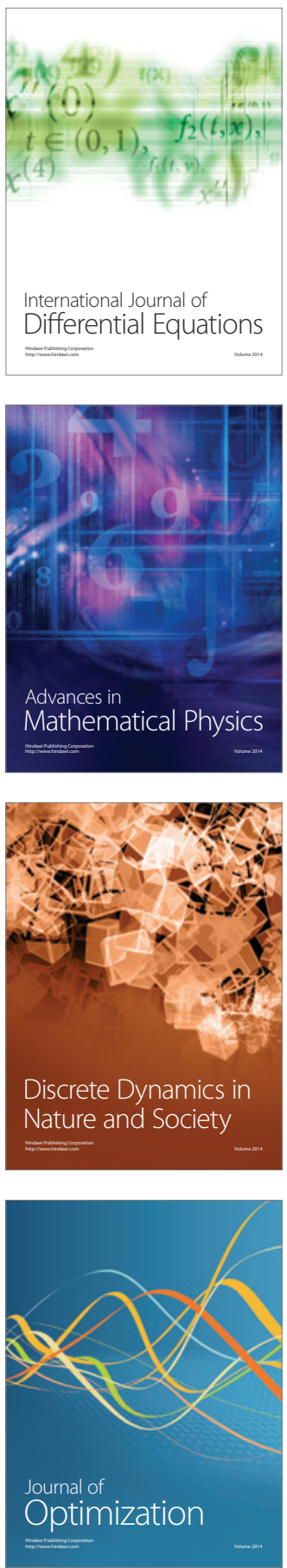